\title{
Micro-buckling of periodically layered composites in regions of stress concentration
}

\author{
Poulios, Konstantinos; Niordson, Christian Frithiof
}

Published in:

Composite Structures

Link to article, DOI:

10.1016/j.compstruct.2016.08.030

Publication date:

2016

Document Version

Peer reviewed version

Link back to DTU Orbit

Citation $(A P A)$ :

Poulios, K., \& Niordson, C. F. (2016). Micro-buckling of periodically layered composites in regions of stress concentration. Composite Structures, 157, 424-435. https://doi.org/10.1016/j.compstruct.2016.08.030

\section{General rights}

Copyright and moral rights for the publications made accessible in the public portal are retained by the authors and/or other copyright owners and it is a condition of accessing publications that users recognise and abide by the legal requirements associated with these rights.

- Users may download and print one copy of any publication from the public portal for the purpose of private study or research.

- You may not further distribute the material or use it for any profit-making activity or commercial gain

- You may freely distribute the URL identifying the publication in the public portal

If you believe that this document breaches copyright please contact us providing details, and we will remove access to the work immediately and investigate your claim 


\title{
Micro-buckling of periodically layered composites in regions of stress concentration
}

\author{
Konstantinos Poulios* and Christian F. Niordson ${ }^{\dagger}$ \\ Department of Mechanical Engineering, Technical University of Denmark \\ Nils Koppels Allé, Building 404, 2800 Kgs. Lyngby, Denmark.
}

September 11, 2016

\begin{abstract}
Under longitudinal compressive loading, periodically layered composites are prone to microbuckling, which is often the decisive mechanism determining their strength. This paper is concerned with the load carrying capacity of structures made of layered composites with respect to micro-buckling related failure in regions of stress concentrations. A series of parametric studies show the effect of non-uniform stress distributions due to bending loads and the presence of geometrical features such as notches and holes on the initiation of micro-buckling. The contribution of the bending stiffness of the reinforcing layers on the resistance against micro-buckling introduces a dependence on the layer thickness, resulting in size-scale dependent strength limits. Therefore, both the shape and dimensions of the considered geometrical features and the layering thickness of the micro-structure are varied as part of the parametric studies. Moreover, the impact of imperfections in the composite micro-structure on the strength of the considered specimens is investigated.
\end{abstract}

Keywords: composite materials, micro-buckling, stress concentration, size effects

\section{Introduction}

Composite materials represent a very attractive option in mechanical design, due to their -in many cases- excellent strength and stiffness to weight performance. Nevertheless, it is well understood how the compressive strength in the direction parallel to the reinforcement of unidirectionally reinforced composites as well as the in-plane compressive strength of periodically layered composites are limited due to micro-buckling and subsequent formation of kink-bands (Fleck, 1997). For this reason, compressive loads parallel to the reinforcement of unidirectionally reinforced composites are undesirable, but unfortunately they are not always possible to avoid. Even if at global level only tensile and bending loads are applied, unfavorable compressive loads occurring locally are often the decisive factor determining the load capacity of a structure, which can be limited further due to stress concentration in critical regions.

A large number of papers have addressed the initiation of compressive load instabilities in unidirectionally reinforced and layered composite materials. In the classical work of Rosen (1965), such

\footnotetext{
${ }^{*}$ E-mail: kopo@mek.dtu.dk

${ }^{\dagger}$ E-mail: cn@mek.dtu.dk
} 
instabilities are divided into shear-mode micro-buckling, where the reinforcing layers or fibers remain parallel and cause shear in the matrix material in-between and the so-called transverse- or extensionalmode micro-buckling, where neighboring fibers periodically converge and diverge, causing periodical transverse compression and tension of the matrix layer. Additionally, micro-buckling can be characterized either as plastic or elastic, depending on whether plastic yielding of the matrix material is involved in the instability initiation or not. Budiansky (1983) and Budiansky and Fleck (1993) have provided simple analytical criteria, that cover both the elastic and imperfection-free solution of Rosen (1965), but also plastic micro-buckling of composites with perfectly elasto-plastic and Ramberg-Osgood matrix materials respectively. The elastic micro-buckling initiation depends on the fiber volume fraction and the shear modulus of the matrix phase, while plastic micro-buckling initiation depends mainly on the ratio between the fiber misalignment angle in the micro-structure and the shear yield strain for the matrix material, but possibly also on the Ramberg-Osgood hardening exponent.

More elaborate but still analytical expressions for imperfection-free elastic micro-buckling are found in (Parnes and Chiskis, 2002) and (Aboudi and Gilat, 2006). Parnes and Chiskis (2002) provided a detailed review of previous and more classical works on the subject and showed that under the applied assumptions, shear-mode micro-buckling is the predominant mode and the transverse mode is associated with higher energy levels. The examples studied in the present paper are also limited to the case of shear-mode micro-buckling. Aboudi and Gilat (2006) exploited an analogy between the buckling initiation and wave propagation equations and obtained corresponding buckling criteria on the basis of exact and approximate dispersion relations for the two and three-dimensional cases respectively.

Plastic micro-buckling and the subsequent formation of kink-bands have been studied both experimentally and numerically. Fleck and Shu (1995) studied the compressive strength of composite samples with different kinds and magnitudes of imperfections based on a homogenized finite-element model which accounts for the fiber bending stiffness. Kyriakides et al. (1994) demonstrated the formation of kink-bands both experimentally and theoretically using a finite-element model with individually discretized fibers. Jensen and Christoffersen (1997) studied the effect of different fiber and matrix materials on the kink-band initiation stress by means of a homogenized model that relies directly on the constitutive laws of the individual constituents.

The aforementioned investigations basically refer to simple geometries and a uniform far-field stress. Additional complexity is introduced when studying the impact of free edges and geometrical features leading to stress concentration. Waas et al. (1990) studied free edge effects in the context of elastic micro-buckling and imperfection-free micro-structures. Their theoretical model predicts that for rather dilute composites, free edge effects can lead to a lower micro-buckling limit than the one predicted by Rosen (1965). However, the case of stress gradients due to geometrical features such as notches is of higher relevance for practical applications.

Stress concentrations in the vicinity of circular holes contained in long fiber reinforced composite plates is an extensively studied case, also in the context of compressive loading. Experiments by Soutis and Fleck (1990), for multi-directional laminates, as well as by Khamseh and Waas (1992), for a uni-ply system, showed that the compressive strength of perforated composite plates increases as the hole diameter decreases, not only in the range where the hole size affects the remaining cross sectional area significantly but also for holes of insignificant size compared to the plate width. In the latter case, the compressive strength dependence on the hole diameter is due to micro-mechanical size effects, which have been subject to several modeling efforts. Soutis and Fleck (1990) and Soutis et al. (1991) used fracture mechanics for capturing this size dependence by interpreting a propagating kink-band as a cohesive crack. Based on experimental evidence, they considered that a stable kinkband exists prior to ultimate failure and propagates up to a critical length, comparable to the hole 
diameter, where the kink-band propagation becomes unstable and the specimen load capacity has been reached. The suggested fracture mechanics based model predicted a size-scale dependent transition from stable to unstable kink-band propagation, leading to the anticipated size-scale dependence of the compressive load capacity. Following a similar fracture mechanics based approach, Bažant et al. (1999) studied the size-scale dependent compressive failure of single edge notched beam specimens as well as the initiation of kink-bands from the smooth surface of notch-free specimens in the presence of compressive stress gradients. Emphasis was put on the deterministic nature of size effects, in contrast to statistical or Weibull-type size effects which are due to the different probabilities of defects present in small and large specimens.

Although the aforementioned fracture mechanics based models introduce a size-scale dependence through the definition of a fracture energy associated with the release of energy in a kink-band, they do not treat the bending stiffness of the reinforcing layers as the root of any size effects explicitly, as in (Fleck and Shu, 1995) and the present paper. In contrast to these fracture mechanics based works, the examples included in the present paper consider the load carrying capacity of notched specimens defined at the point of micro-buckling initiation without accounting for any subsequent propagation of the formed kink-band, even if this could in some cases lead to a higher ultimate load before global failure. The reported results demonstrate a size-scale dependence of the load carrying capacity even without considering a pseudo-ductile behavior prior to ultimate failure.

The effect of the fiber bending stiffness is inherently included in the local-global model approach suggested by Waas et al. (1998) for predicting the compressive strength of a notched unidirectionally reinforced composite. The local model used there represents a rectangular region of interest consisting of individual fibers and layers of the matrix material, which are explicitly discretized by means of the finite-element method. Due to the fiber diameter typically being orders of magnitude smaller than the dimensions of the considered components or test specimens, only a small region of interest could be modeled. The boundary conditions for this small region were linked to a global finite-element model, where the reinforcement is captured at the macro-scale simply through an orthotropic material law. In a variation of this approach, Ahn and Waas (2002) linked the local model to an analytical farfield solution instead of a global finite-element model. The local-global model approach was also included in the effort by Davidson et al. (2013) to create a unified predictive model with respect to both tensile and compressive open hole strengths of composite laminates. The lack of bidirectional coupling between the local and global models was addressed in a recent development by Wind et al. (2015), who embedded the local finite-element model in the global one by means of the superelement approach. In that work, the numerical model was used in combination with experimental testing in order to investigate the failure of notched specimens loaded in a four point bending setup. Compared to the explicit discretization of the micro-structure within a micro-region of interest followed in the aforementioned works, the present work demonstrates the use of a homogenized model in order to capture local phenomena such as micro-buckling directly in the global model.

The present paper relies on extensive numerical studies, for investigating the effect of stress gradients on the initiation of micro-buckling. Emphasis is put on the effect of size dependent failure limits due to the contribution of the bending stiffness of the reinforcing layers to the material resistance against micro-buckling. Moreover, the effects of stress concentration and misalignment imperfections are studied in detail. It should be noted, that failure is defined here as the initiation of a local instability without intending to study the subsequent formation and propagation of kink-bands in detail. For this reason, any post-buckling results presented must be interpreted in the context of the underlying assumptions of perfect bonding and absence of reinforcement and matrix damage. Moreover, a study of compressive instabilities initiated by fiber-matrix splitting as modeled analytically by Lee and Waas (1999) and numerically by Prabhakar and Waas (2013), is beyond the scope of this paper. 
The paper is organized in six sections. Following this introduction, Section 2 outlines the numerical model used for all parametric studies to be presented in the subsequent sections. Section 3 includes the bending of a plain beam as a reference case, presenting the basic form of the observed kink-bands and showing the impact of the reinforcing layer thickness as well as the impact of assumed waviness imperfections on the compressive strength. Section 4 presents results for a notched beam subjected to bending, while Section 5 presents numerical results of kink-bands initiated around a circular hole of a specimen subjected to compression parallel to its reinforcement. Section 6 summarizes and concludes the paper.

\section{Numerical model}

All parametric studies presented in the following sections were carried out by means of the homogenized finite-element model by Poulios and Niordson (2016). The main features of this model are outlined in this section. The model represents a layered micro-structure consisting of an elastic phase combined with a more compliant and plastically deformable phase. Both phases are assumed to be subjected to plane strain conditions. In analogy to the three-dimensional micro-structure of a unidirectionally reinforced fiber composite, the material of the strong phase is denoted by convention as the fiber material while the weak phase is referred to as the matrix material. The volumetric content of each phase is an input to the model but it can be variable across the simulated domain, defined e.g. node-wise on a finite-element mesh. The orientation of the micro-structure is a further input for the model and can also be spatially varying.

Due to the applied homogenization, it is not necessary to discretize the material micro-structure with its individual phases explicitly. In contrast to most other homogenized models however, the applied model utilizes the constitutive laws of the individual constituents directly, rather than involving a constitutive law for the composite material. Rate-independent hyper-elasticity is assumed for the reinforcing phase and rate-independent hyper-elasto-plasticity is assumed for the matrix material. Different hardening behaviors such as linear, exponential or Ramberg-Osgood hardening can be associated with the matrix material.

The considered homogenized model accounts for the relative deformation between the reinforcement and the matrix through a two-dimensional internal kinematic variable $\boldsymbol{d}$. Fig. 1 shows possible micro-structure configurations corresponding to different values of $\boldsymbol{d}$ for the same undeformed homogenized unit-cell (dashed green line). In total the unknown quantities to solve for in each load step of the quasi-static simulation are the displacements field $\boldsymbol{u}$, the internal kinematic variable field $\boldsymbol{d}$ and the field $\xi_{m}$ which is a scaled version of the effective plastic strain increment for the matrix material. All aforementioned unknown fields are approximated by means of 8-node quadrilateral elements, resulting in a finite-element model with five degrees of freedom per node.

The basic idea behind the model is that two distinct deformation tensors for the fiber and the matrix phases can be calculated based on the deformation gradient $\boldsymbol{F}_{u}$ of the homogenized displacements field $\boldsymbol{u}$ and the internal kinematic variable $\boldsymbol{d}$, respectively as:

$$
\begin{gathered}
\tilde{\boldsymbol{F}}_{f}\left(\chi_{N}\right)=\boldsymbol{F}_{u}-\frac{1}{c_{f}} \boldsymbol{d} \boldsymbol{N}^{T}-\chi_{N} \frac{1}{c_{f}} \nabla \boldsymbol{d}\left(\boldsymbol{I}-\boldsymbol{N} \boldsymbol{N}^{T}\right) \text { and } \\
\boldsymbol{F}_{m}=\boldsymbol{F}_{u}+\frac{1}{c_{m}} \boldsymbol{d} \boldsymbol{N}^{T},
\end{gathered}
$$

where $\chi_{N}$ is the distance from the mid-plane of the fiber layer, $N$ is the unit vector perpendicular to the micro-structure layers in the undeformed configuration, while $c_{f}$ and $c_{m}$ are the volumetric fractions of the fiber and matrix layers respectively, with $c_{f}+c_{m}=1$. 

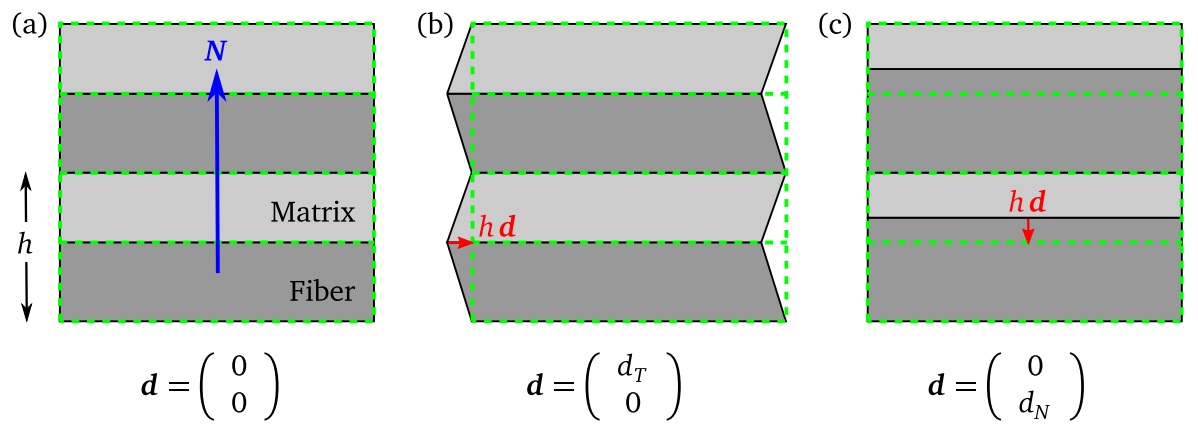

Figure 1: Deformation states of the composite micro-structure corresponding to different values of the internal kinematic variable $\boldsymbol{d}$.

In order to account for plasticity in the matrix material, the following multiplicative split of the deformation tensor $\boldsymbol{F}_{m}$ into an elastic part $\boldsymbol{F}_{m}^{e}$ and a plastic part $\boldsymbol{F}_{m}^{p}$ is considered:

$$
\boldsymbol{F}_{m}=\boldsymbol{F}_{m}^{e} \boldsymbol{F}_{m}^{p} .
$$

Based on this, $\mathrm{J}_{2}$-flow plasticity for the matrix material leads to the following closed-form expression for the elastic deformation in the matrix, in terms of the corresponding logarithmic strain tensor:

$$
\ln \left(\boldsymbol{F}_{m}^{e} \boldsymbol{F}_{m}^{e T}\right)=\ln \left(\boldsymbol{F}_{m} \boldsymbol{C}_{m *}^{p}{ }^{-1} \boldsymbol{F}_{m}{ }^{T}\right)-\xi_{m} \operatorname{dev}\left(\ln \left(\boldsymbol{F}_{m} \boldsymbol{C}_{m *}^{p}{ }^{-1} \boldsymbol{F}_{m}{ }^{T}\right)\right)
$$

where the inverse right Cauchy-Green strain tensor $\boldsymbol{C}_{m *}^{p}{ }^{-1}$ represents the known plastic deformation in the previous time step, while the unknown scalar $\xi_{m}$ indirectly determines the plastic strain increment during the current step. The current cumulative plastic strain $\gamma_{m}$ in the matrix material can be evaluated from the corresponding value $\gamma_{m *}$ in the previous time step based on the following update formula:

$$
\gamma_{m}=\gamma_{m *}+\xi_{m}\left\|\operatorname{dev}\left(\ln \left(\boldsymbol{F}_{m} \boldsymbol{C}_{m *}^{p}{ }^{-1} \boldsymbol{F}_{m}{ }^{T}\right)\right)\right\| \text {. }
$$

For further details the reader is referred to (Poulios and Niordson, 2016).

The elastic energy density in the matrix material is expressed by means of the following hyperelastic constitutive law:

$$
\psi_{m}^{e}=\frac{K_{m}}{8}\left(\ln \left|\boldsymbol{F}_{m}^{e} \boldsymbol{F}_{m}^{e T}\right|\right)^{2}+\frac{G_{m}}{4}\left\|\operatorname{dev}\left(\ln \left(\boldsymbol{F}_{m}^{e} \boldsymbol{F}_{m}^{e T}\right)\right)\right\|^{2} .
$$

In the case of the fiber layers, recalling that $\tilde{\boldsymbol{F}}_{f}$ is a function of $\chi_{N}$, the elastic energy density can be expressed with the help of a similar hyper-elastic constitutive law as the following average through the fiber height $h_{f}$ :

$$
\psi_{f}^{e}=\frac{1}{h_{f}} \int_{-h_{f} / 2}^{h_{f} / 2}\left(\frac{K_{f}}{8}\left(\ln \left|\tilde{\boldsymbol{F}}_{f} \tilde{\boldsymbol{F}}_{f}^{T}\right|\right)^{2}+\frac{G_{f}}{2}\left(\left|\tilde{\boldsymbol{F}}_{f}\right|^{-2 / 3} \operatorname{Tr}\left(\tilde{\boldsymbol{F}}_{f} \tilde{\boldsymbol{F}}_{f}^{T}\right)-3\right)\right) d \chi_{N} .
$$

The choice of a constitutive law in Eq. (7) that does not involve the matrix logarithm operator in the deviatoric part, allows an analytical approximate evaluation of the integral based on a linearization of the integrand with respect to point $\chi_{N}=0$. This analytical integration leads to an elastic energy expression that includes a term with $\nabla \boldsymbol{d}$ multiplied by a coefficient proportional to the square of the characteristic length parameter $h_{f}$. 
The total elastic energy density can then be defined as:

$$
\psi^{e}=c_{f} \psi_{f}^{e}+c_{m} \psi_{m}^{e},
$$

which can be used in a classical hyper-elasto-plasticity modeling approach to express the final equilibrium equations through a virtual work expression in terms of $\nabla \boldsymbol{u}, \boldsymbol{d}, \nabla \boldsymbol{d}$ and $\xi_{m}$ as well as the variations $\nabla \delta \boldsymbol{u}, \delta \boldsymbol{d}$ and $\nabla \delta \boldsymbol{d}$. An additional equation needed for solving for $\xi_{m}$ is provided by fulfilling a plastic yield criterion with respect to a yield surface $f$, as:

$$
\begin{cases}f\left(\nabla \boldsymbol{u}, \boldsymbol{d}, \xi_{m}\right)=0 & \text { : if } f \geq 0 \\ \xi_{m}=0 & \text { : if } f<0\end{cases}
$$

A standard Galerkin procedure is employed in order to enforce Eq. (9) in a weak sense, while further details on the performed discretization can be found in (Poulios and Niordson, 2016).

All numerical simulations presented in subsequent sections assume $\mathrm{J}_{2}$-flow plasticity with a RambergOsgood hardening law, corresponding to the following definition of the yield surface:

$$
f\left(\nabla \boldsymbol{u}, \boldsymbol{d}, \xi_{m}\right)=\left\|\operatorname{dev}\left(\boldsymbol{\sigma}_{m}\right)\right\|-\sqrt{\frac{2}{3}} \sigma_{y m}\left(\sqrt{\frac{2}{3}} \frac{E_{m}}{\alpha_{m} \sigma_{y m}} \gamma_{m}\right)^{1 / n_{m}},
$$

where the Cauchy stress tensor in the matrix material $\boldsymbol{\sigma}_{m}$ can be derived as a closed-form function of $\nabla \boldsymbol{u}, \boldsymbol{d}$ and $\xi_{m}$ through differentiation of Eq. (6), while the cumulative plastic strain $\gamma_{m}$ is already defined as a function of $\nabla \boldsymbol{u}, \boldsymbol{d}$ and $\xi_{m}$ through Eq. (5). The constants $\sigma_{y m}, \alpha_{m}$ and $n_{m}$ are the common Ramberg-Osgood parameters for the matrix material.

Although the aforementioned constitutive laws are non-linear, it is useful for dimensional analysis purposes to consider the initial plane strain Young's moduli of the two materials as reference values, defined as:

$$
\bar{E}_{f}=\frac{E_{f}}{1-v_{f}^{2}} \quad \text { and } \quad \bar{E}_{m}=\frac{E_{m}}{1-v_{m}^{2}},
$$

where $E_{f}, E_{m}$ and $v_{f}, v_{m}$ are the Young's moduli and Poisson's ratios of the two materials which are directly linked to the initial bulk and shear moduli $K_{f}, K_{m}$ and $G_{f}, G_{m}$ which appear in Eqs. (6) and (7).

\section{Bending of a plain beam}

Fig. 2 shows the mesh and boundary conditions used for studying the bending of a reinforced plain beam with a periodic layered micro-structure nominally parallel to the horizontal direction. The default values of the fiber volume fraction and the height of each reinforcing layer are $c_{f}=0.587$ and $h_{f}=0.0052 \mathrm{~mm}$, respectively. The nominal height of the considered beam specimen is $H=3.867 \mathrm{~mm}$ and its length is $L=4 \cdot H$. All results presented in this section refer to a unit reference thickness $T$ in the out of plane direction. The default mesh, shown in Fig. 2 , includes a finer meshed region with dimensions $0.5 \mathrm{H} \times 0.3 \mathrm{H}$ divided into elements of size equal to $0.021 \mathrm{~mm} \times 0.028 \mathrm{~mm}$. In order to trigger micro-buckling to occur in the central and finer meshed region, the top surface is shifted vertically by:

$$
\delta y=\left\{\begin{array}{cl}
H_{\delta}(1-\cos (2 \pi x / H)) / 2 & \text { for }|x| \leq H / 2 \\
H_{\delta} & \text { for }|x|>H / 2
\end{array},\right.
$$




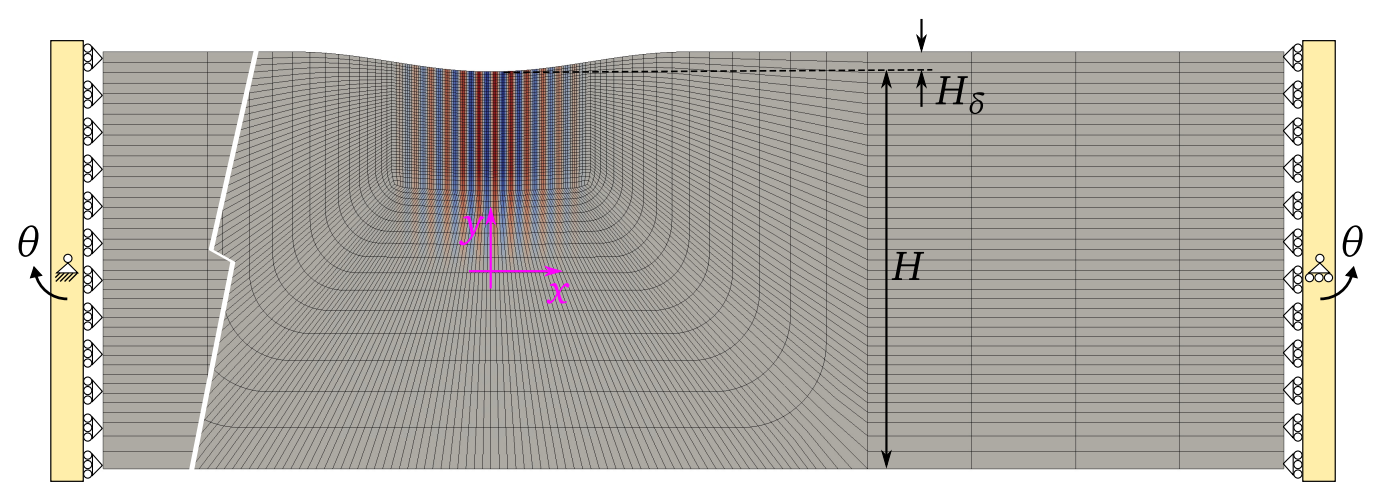

Figure 2: Mesh and boundary conditions for the plain beam bending example.

resulting in a slightly increased height towards the beam ends. For the sake of illustration, the geometry shown in Fig. 2 corresponds to $H_{\delta}=0.05 \cdot \mathrm{H}$ but a much lower value of $H_{\delta}=0.001 \cdot \mathrm{H}$ is considered as the default for the results to be presented, unless otherwise stated.

Additional to the aforementioned geometrical imperfection, a sinusoidal waviness of the reinforcing layers is assumed within the area of interest, with peak-to-peak amplitude $a_{w}$ and wavelength $p_{w}$ varied as part of the present study. The color plot embedded in Fig. 2 illustrates the fiber orientation angle in the region of the prescribed imperfection, corresponding to the default value for the imperfection wavelength $p_{w}=30 \cdot h_{f}=0.156 \mathrm{~mm}$. The illustrated fiber waviness imperfection is symmetric with respect to the $y$-axis, but a corresponding asymmetric imperfection can easily be obtained by a phase shift of $\pi / 2$.

In total, the geometric characteristics of the considered system can be described by means of the following dimensionless parameters:

- The relative beam height variation $\bar{H}_{\delta}=H_{\delta} / H$.

- The relative fiber height $\bar{h}_{f}=h_{f} / H$.

- The relative fiber waviness wavelength $\bar{p}_{w}=p_{w} / h_{f}$.

- The maximum misalignment angle $\bar{\phi} \approx \pi a_{w} / p_{w}$ in radians.

In terms of its material properties, the composite can be characterized through the dimensionless parameters $c_{f}, \bar{E}_{f} / \bar{E}_{m}, v_{f}, v_{m}, \sigma_{y m} / \bar{E}_{m}, n_{m}$ and $\alpha_{m}$. The material parameters considered in the present example are $E_{f}=27600 \mathrm{MPa}, v_{f}=0.3, E_{m}=1478.3 \mathrm{MPa}, v_{m}=0.38, \sigma_{y m}=90 \mathrm{MPa}$, $n_{m}=4$ and $\alpha_{m}=3 / 7$. These correspond to a moderate value of the fiber-to-matrix contrast parameter $\bar{E}_{f} / \bar{E}_{m}=63.6$ and a relative yield limit on the order of $2 \%$, i.e. $\sigma_{y m} / \bar{E}_{m}=0.0189$.

In line with the above dimensionless description of the system, a normalized bending stress is introduced as a dimensionless measure of the applied bending moment $M$ :

$$
\varepsilon_{b}=\frac{\sigma_{b}}{\bar{E}}=\frac{6 M /\left(T H^{2}\right)}{c_{f} \bar{E}_{f}+\left(1-c_{f}\right) \bar{E}_{m}} .
$$

The strain like quantity $\varepsilon_{b}$ corresponds to the maximum compressive and tensile strains that would occur close to the top and bottom surfaces of the beam under the bending moment $M$, assuming a linear distribution of the stresses through the beam height $H$ and a linear material behavior with the weighted average Young's modulus $\bar{E}$. 
In the considered example, the loading of the beam is effected by prescribing a rotation angle $\theta$ at the beam ends, as indicated in Fig. 2. Higher order boundary conditions corresponding to the variable $\boldsymbol{d}$ prevent any deformation of the fibers with respect to the matrix at the beam ends, i.e. both modes of Fig. 1 b) and c) are restricted. As the angle $\theta$ increases, the compressive stresses in the upper part of the beam also increase up to the point that a micro-buckling instability is triggered. In order to track both the instability initiation and the post-buckling behavior, numerical continuation with respect to $\theta$ is performed according to Ligursky and Renard (2014).

Fig. 3 shows the evolution of the applied bending load versus the prescribed angle $\theta$ for the reference value $\bar{H}_{\delta}=0.001$ as well as for two alternative geometries with considerable beam height variations $\bar{H}_{\delta}=0.05$ and $\bar{H}_{\delta}=0.15$. The three curves exhibit a characteristic form for buckling simulations with a linear evolution up to the critical bending load (point A), where micro-buckling initiates, a subsequent unstable unloading branch and a final post-buckling loading region at a considerably lower bending load.

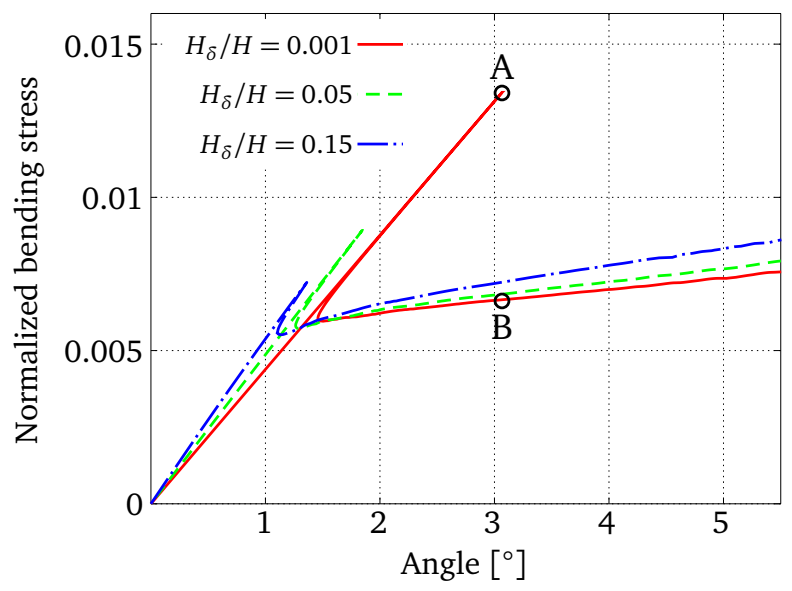

Figure 3: Bending load evolution for different beam height variations $H_{\delta}$ (for $c_{f}=0.587, \bar{h}_{f}=0.0013$, $\bar{p}_{w}=30, \bar{\phi}=0.60^{\circ}$, asymmetric waviness imperfection).

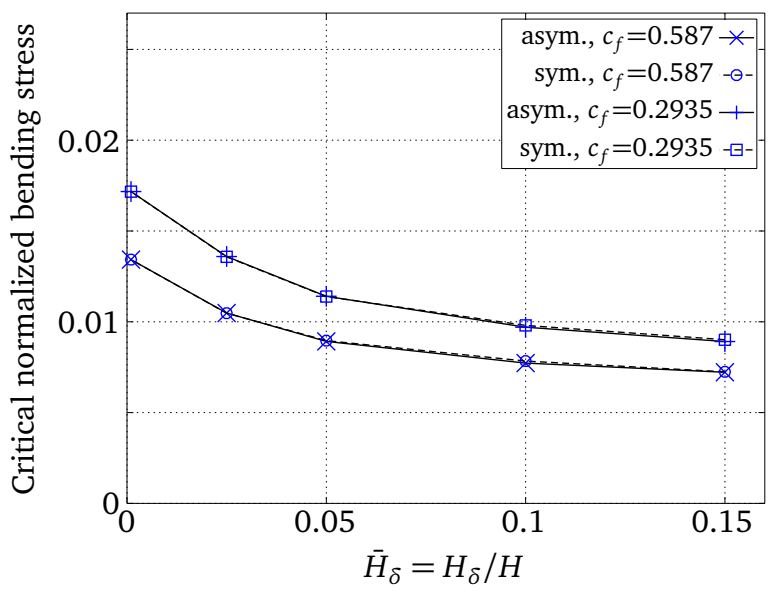

Figure 4: Critical bending load as a function of the beam height variation $H_{\delta}$ (for $\bar{h}_{f}=0.0013, \bar{p}_{w}=30$, $\bar{\phi}=0.60^{\circ}$ ). 
The dependence of the critical bending load on the geometrical imperfection $H_{\delta}$ is further illustrated in Fig. 4, which also includes results for the fiber volume fraction $c_{f}$ reduced to the half of its reference value. It should be noted that although the critical normalized bending stress for the lower fiber volume fraction is higher, due to the lower reference Young's modulus $\bar{E}$, the absolute value of the applied bending moment $M$ is lower, as would be expected. Despite the strong dependence of the critical bending load on $\bar{H}_{\delta}$, the curves shown in Fig. 4 indicate that the default value of 0.001 is sufficiently low for the corresponding results to be considered equivalent to the plain beam with $H_{\delta} \rightarrow 0$.

Apart from the default asymmetric waviness imperfections, Fig. 4 also includes results for a symmetric one, but the impact of the phase shift between these two kinds of imperfection on the critical bending load is minimal. Nevertheless, a comparison between Figs. 5 and 6 shows important differences in the post-buckling deformation of the beam. For the asymmetric waviness imperfection, the results shown on the left hand side of Fig. 5 correspond to the initiation of micro-buckling (point A in Fig. 3), while the load step shown on the right hand side corresponds to approximately the same bending angle $\theta$ but within the post-buckling region (point B in Fig. 3). The grey-scale map in the background illustrates the assumed fiber waviness misalignment angle near the top surface. At micro-buckling initiation (left hand side picture) a limited amount of plastic strain on the order of $3 \%$ is observed for the matrix material in regions of high fiber misalignment but there is no visible indication of the imminent instability. The initiation of micro-buckling is identified clearly only when looking at the velocity field. The velocity profile of the top surface indicates the location, form and width of a bulge that has begun to form.

The picture on the right hand side of Fig. 5 shows the fully formed bulge and the severe plastic deformation in the matrix material under the assumption of ductile behavior. The affected conical shape region in the interior of the beam is formed by a pair of kink-bands of increasing width towards the top surface. As the bending angle $\theta$ increases, the kink-bands broaden to accommodate the angle increase and the damage cone propagates deeper into the material. The observed formation is similar to the one found experimentally and verified numerically by Liu et al. (2014), for an ultra high molecularweight polyethylene fiber in polyurethane matrix composite. In that work, the term "bending hinge" was used for characterizing the observed double kink-band cone formation. It is essential to highlight that the observed cone does not necessarily need to extend through the complete beam height and form a perfect hinge. In the present simulations the cone starts with a small but finite depth which increases in the unstable branch of the displacement controlled simulation. Of course, the unstable path followed in numerical continuation is an idealization of the snap-back that will occur between points A and B dynamically. It should also be noted that more common composite systems such as glass or carbon fibers in an epoxy resin matrix, can not reach levels of deformation like on the right hand side of Fig. 5, without other failure mechanism such as matrix cracking, fiber-matrix splitting or fiber breakage to occur first, as demonstrated e.g. in (Pinho et al., 2012).

Fig. 6 shows corresponding snapshots at buckling initiation and in the post-buckling region for the case with a symmetric waviness imperfection. Due to perfect symmetry, two bulges start to form as seen on the left hand side but the corresponding cones can not grow unhindered as in the previous case. The collision between the two cones results into the more complex formation shown on the right hand side of Fig. 6 .

Fig. 7 shows the dependence of the critical bending load on the fiber relative height $\bar{h}_{f}$, which demonstrates a significant size effect. For example if the beam height is reduced to 100 times the fiber height, or equivalently if the fiber height is increased to $1 / 100$-th of the beam height, an increase in the bending load capacity of the beam in the order of $60 \%$ is predicted, compared to the limit $\bar{h}_{f} \rightarrow 0$. The graph also indicates that the default value $\bar{h}_{f}=0.0013$ used in the rest of the parametric studies 

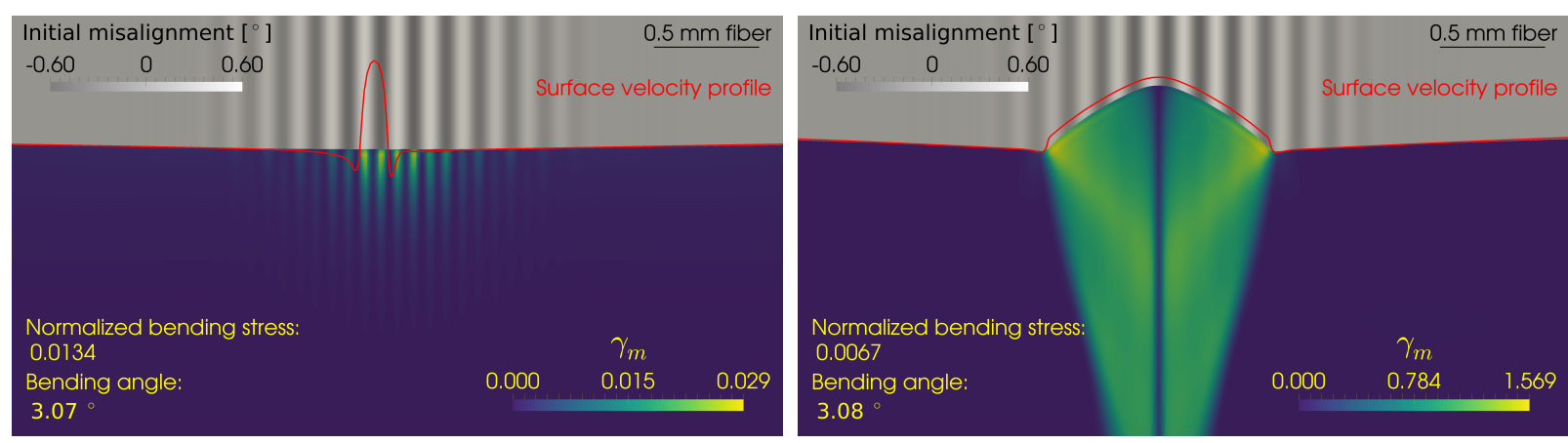

Figure 5: Micro-buckling initiation and formation of kink-bands (for $c_{f}=0.587, \bar{H}_{\delta}=0.001, \bar{h}_{f}=$ $0.0013, \bar{p}_{w}=30, \bar{\phi}=0.60^{\circ}$, asymmetric waviness imperfection).
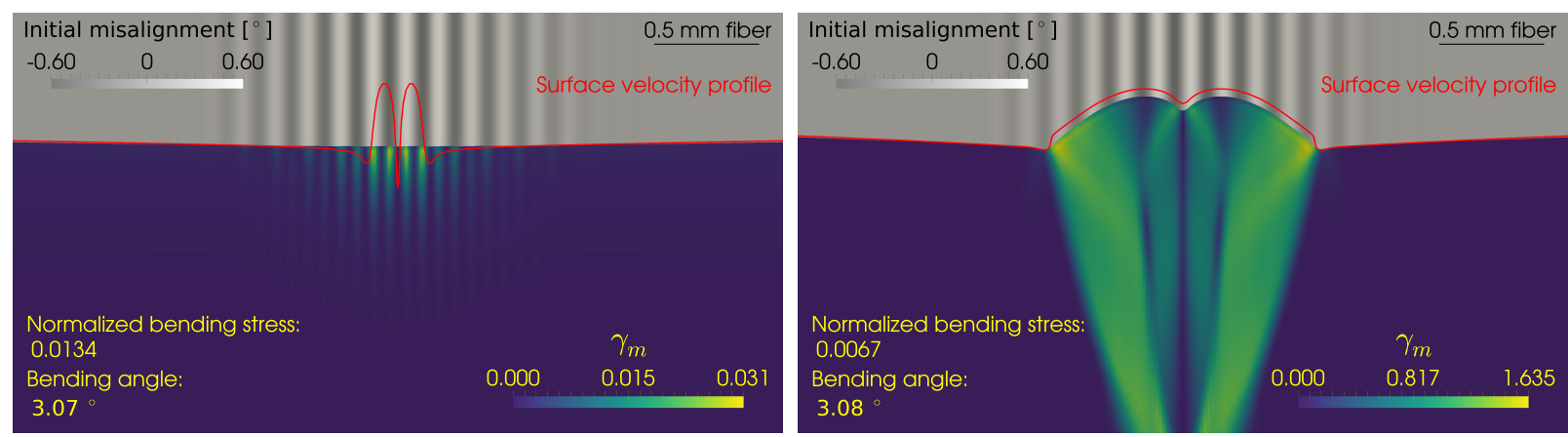

Figure 6: Micro-buckling initiation and formation of kink-bands (for $c_{f}=0.587, \bar{H}_{\delta}=0.001, \bar{h}_{f}=$ $0.0013, \bar{p}_{w}=30, \bar{\phi}=0.60^{\circ}$, symmetric waviness imperfection).

corresponds to a moderate size-scale effect of about $8 \%$.

Fig. 8 illustrates the dependence of the critical bending load on the wavelength $p_{w}$, while the ratio $a_{w} / p_{w}$ and consequently also the misalignment angle $\bar{\phi}$ are kept constant. It appears that independent from the fiber volume fraction, variation of the wavelength ratio in the range from 40 to 120 affects the bending load capacity only marginally. For lower imperfection wavelengths the critical bending load increases due to the contribution of the fiber bending stiffness as predicted also by the classical relationship of Rosen (1965) and discussed also in (Parnes and Chiskis, 2002).

The dependence of the critical bending load on the maximum fiber misalignment angle $\bar{\phi}$ is presented in Fig. 9. The decay of the critical bending load with increasing misalignment angle is expectable. Nevertheless, there is a significant quantitative difference with respect to the predictions of Budiansky and Fleck (1993) for uniform uniaxial compression. The corresponding analytical solution according to Budiansky and Fleck (1993) is included in the plots for comparison. In order to clarify the root of the observed difference between this analytical result for pure compression and the numerical results for the beam bending case, considered here, two further curves are included in the same graph. Numerical simulation of the same composite material under pure compression with a relative fiber waviness wavelength $\bar{p}_{w}=60$ yields results fairly close to the analytical solution. However, reducing the relative wavelength value to $\bar{p}_{w}=30$ results in considerably higher critical bending loads due to the effect discussed in the context of Fig. 8. This indicates the relatively short imperfection wavelength corresponding to $\bar{p}_{w}=30$ as the major factor for the comparatively high critical bending load plotted in Fig. 9 . 


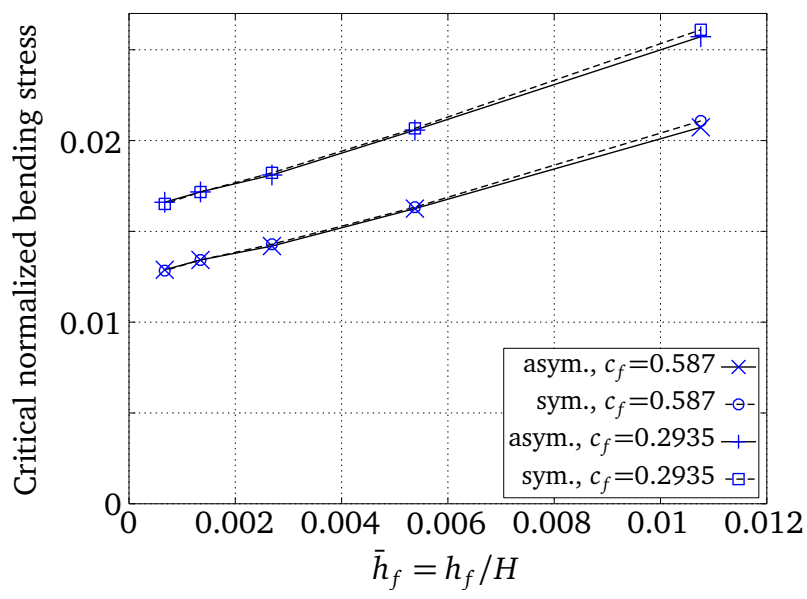

Figure 7: Critical bending load as a function of the fiber height $h_{f}\left(\right.$ for $\left.\bar{H}_{\delta}=0.001, \bar{p}_{w}=30, \bar{\phi}=0.60^{\circ}\right)$.

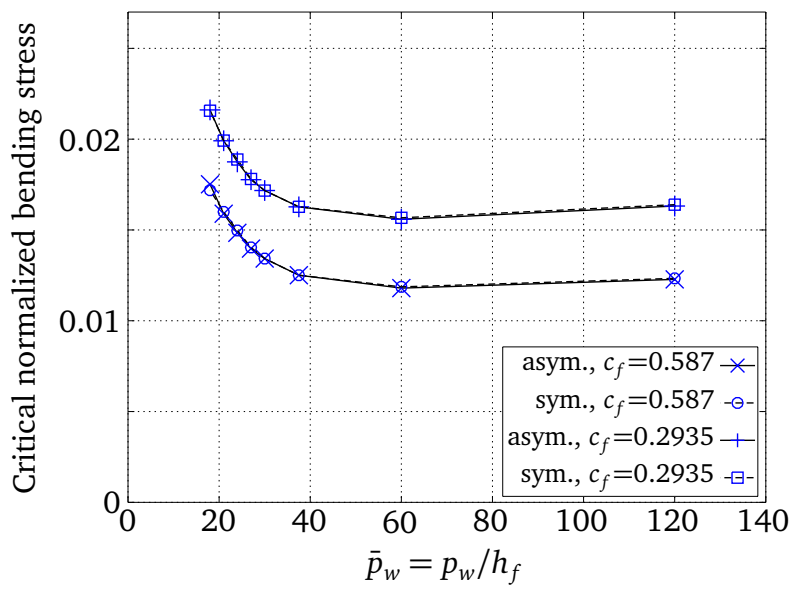

Figure 8: Critical bending load as a function of the fiber waviness wavelength $p_{w}$ (for $\bar{H}_{\delta}=0.001$, $\bar{h}_{f}=0.0013, \bar{\phi}=0.60^{\circ}$ ).

Nevertheless, there are further aspects to consider when comparing the numerical modeling results for bending with analytical solutions for pure compression. The most important factors can be summarized as follows:

a) The considered analytical solution does not account for the fiber bending stiffness, which appears to play a significant role for high values of $\bar{h}_{f}$ and low values of $\bar{p}_{w}$.

b) The non-uniform stress distribution under bending is not directly comparable to the uniform stress field under pure compression.

c) The actual compressive stress close to the top surface may deviate from the normalized bending stress from Eq. (13) because of a non linear stress distribution across the beam height $H$, e.g. due to the presence of a notch.

d) The nonlinear constitutive law, corresponding to Eq. (6), results in a slightly higher effective shear modulus than the initial shear modulus $G_{m}$ used in the evaluation of the analytical solution.

Especially with regard to the third of the above listed factors, further insight will be provided by the numerical example presented in the following section. 

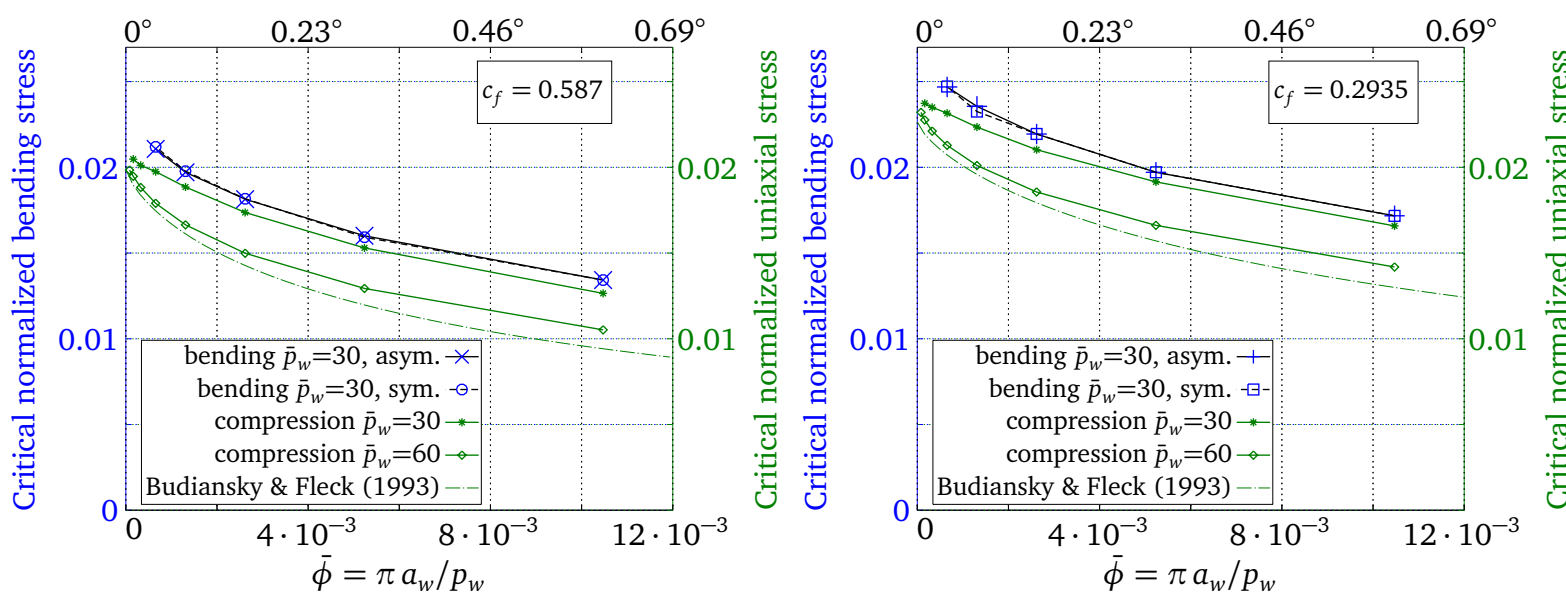

Figure 9: Critical bending load as a function of the fiber waviness amplitude $a_{w}\left(\right.$ for $\bar{H}_{\delta}=0.001, \bar{h}_{f}=$ 0.0013).

\section{Bending of a notched beam}

Another example of a beam subjected to pure bending load is presented in this section. Its distinguishing characteristic compared to the plain beam studied in the previous section is the presence of a V-shaped notch at the center of the beam. The basic geometry, mesh and boundary conditions of the corresponding model are shown in Fig. 10.

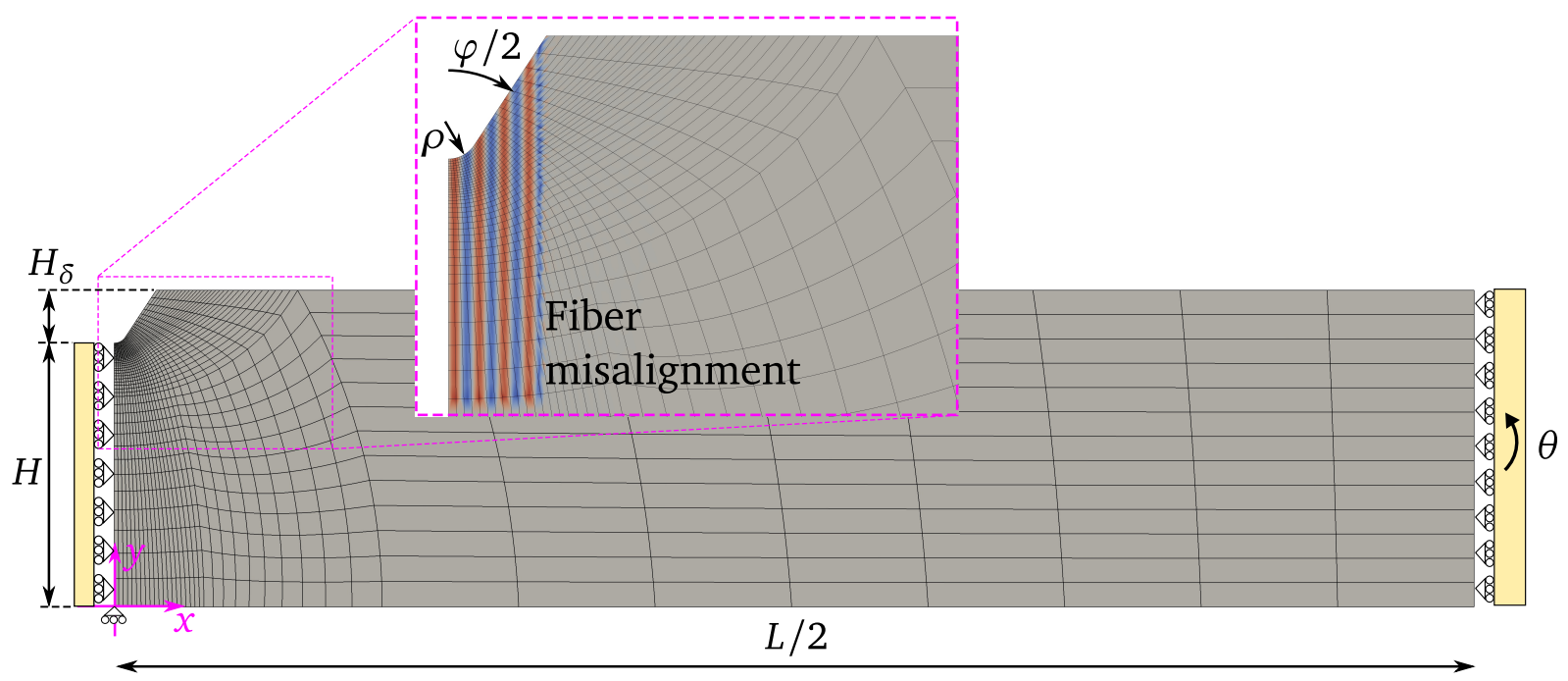

Figure 10: Mesh and boundary conditions for the V-notched beam bending example.

With the reduced height $H$ as a reference length, all further geometrical characteristics of the considered V-notched beam can be described through the length ratios $\bar{H}_{\delta}=H_{\delta} / H$ and $\bar{L}=L / H$, the relative notch tip radius $\bar{\rho}=\rho / H$ and the notch opening angle $\varphi$. In the numerical studies to be presented, $\varphi$ is kept constant and equal to $67^{\circ}$, while all further length ratios are varied, with respect to a fixed reference height $H=3.867 \mathrm{~mm}$, except for the fixed beam length $L=20 \mathrm{~mm}$, which is of minor importance, provided that it is sufficiently large. 
The region close to the notch tip is meshed finer with elements of sizes comparable to the plain beam of the previous section. Moreover, this central region is provided as before with a reinforcement waviness of amplitude $a_{w}$ and wavelength $p_{w}$. The waviness illustrated in the picture embedded in Fig. 10 is symmetric with respect to the y-axis and corresponds to the default wavelength parameter $p_{w}=0.156 \mathrm{~mm}$. It should be noted that numerical results corresponding to an asymmetric waviness are based on a full model, with similar boundary conditions to Fig. 2 instead of the half model of Fig. 10. In total, in the numerical results to follow, the micro-structure is characterized by the same three dimensionless parameters as the plain beam example: The relative fiber height $\bar{h}_{f}=h_{f} / H$, the relative fiber waviness wavelength $\bar{p}_{w}=p_{w} / h_{f}$ and the maximum misalignment angle $\bar{\phi} \approx \pi a_{w} / p_{w}$.

The results of the parametric study indicate the relative fiber height $\bar{h}_{f}$ as the most significant of the parameters affecting the bending load capacity of the beam investigated. Fig. 11 shows the critical bending load as a function of $\bar{h}_{f}$. The load carrying capacity of the notched beam is as expected considerably lower than the corresponding plain beam results of Fig. 7. This difference is, however, more pronounced for small relative fiber height values, starting with a critical bending load on the order of $40 \%$ of the plain beam capacity for the lowest value of $\bar{h}_{f}=6.7 \cdot 10^{-4}$ and increasing up to approximately $80 \%$ of the plain beam capacity for the maximum value of $\bar{h}_{f}=5.4 \cdot 10^{-3}$.
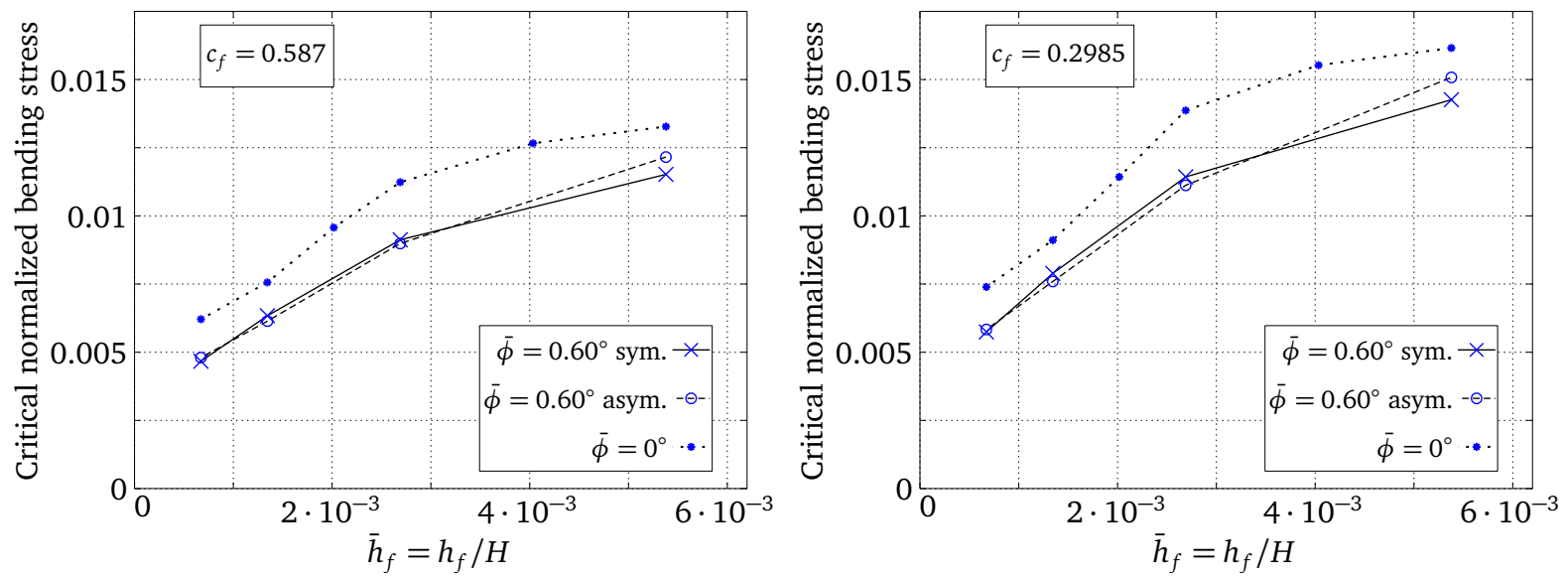

Figure 11: Critical bending load as a function of the fiber height $h_{f}\left(\right.$ for $\left.\bar{H}_{\delta}=0.2, \bar{\rho}=0.0507, \bar{p}_{w}=30\right)$.

Fig. 12 presents the impact of the relative fiber waviness wavelength $\bar{p}_{w}$ on the critical bending load. The form of this graph is very different compared to the corresponding result for the plain beam, shown in Fig. 8. In contrast to the behavior of the plain beam, the bending load capacity of the notched beam increases considerably with increasing $\bar{p}_{w}$. Moreover, there is a significant difference between the critical loads obtained for symmetric and asymmetric waviness imperfections, indicating an important effect of the location of the maximum misalignment angle with respect to the notch location. It appears that an asymmetric waviness imperfection, with its maximum misalignment occurring at location $x=0$, results in a lower bending load capacity in general.

Variation of the waviness amplitude $a_{w}$ at a constant waviness wavelength $p_{w}$ yields the results shown in Fig. 13. Although there is a significant reduction in the critical bending load with increasing $a_{w}$ and misalignment angle $\bar{\phi}$, the effect is less pronounced than in the corresponding case for a plain beam, shown in Fig. 9. Even with the imperfection reduced to zero, the effect of the notch alone is sufficient for micro-buckling to initiate at a load less than $40 \%$ of the corresponding load for a plain beam. 


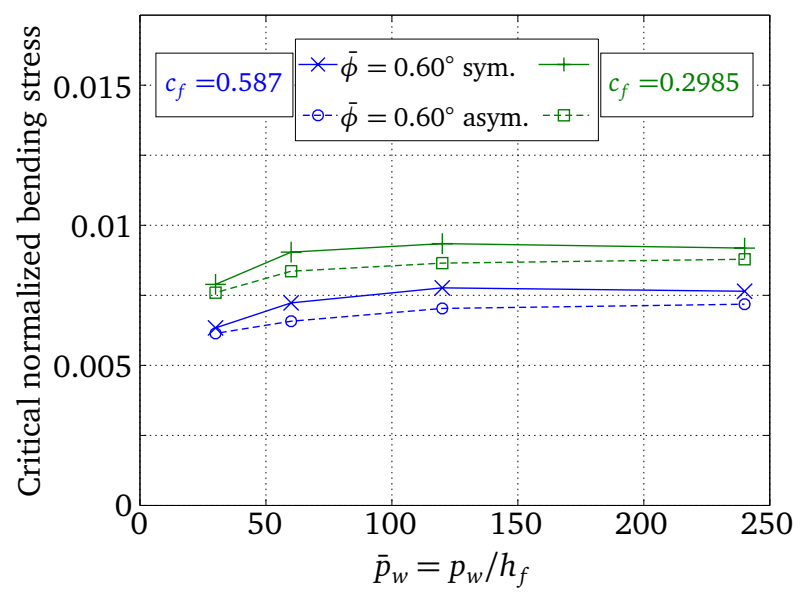

Figure 12: Critical bending load as a function of the fiber waviness wavelength $p_{w}\left(\right.$ for $\bar{H}_{\delta}=0.2, \bar{\rho}=$ 0.0507, $\bar{h}_{f}=0.0013, \bar{\phi}=0.60^{\circ}$ ).

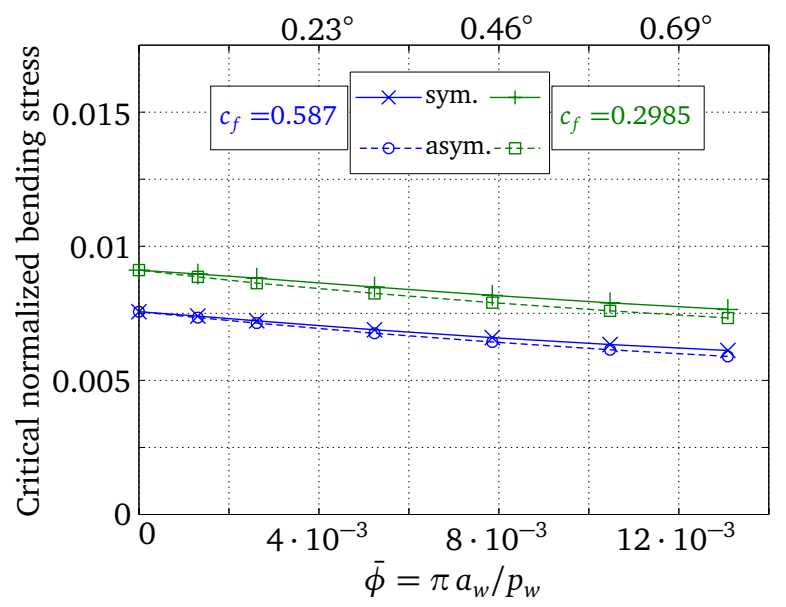

Figure 13: Critical bending load as a function of the fiber waviness amplitude $a_{w}$ (for $\bar{H}_{\delta}=0.2, \bar{\rho}=$ 0.0507, $\left.\bar{h}_{f}=0.0013, \bar{p}_{w}=30\right)$.

Fig. 14 summarizes results from an investigation of the impact of the notch tip radius $\rho$ on the bending load capacity of the beam. Interestingly, the critical bending load seems to depend only weakly on the notch tip radius within a fairly wide range of $\bar{\rho}$ from approximately 0.013 to 0.2 . Local minima and maxima are found in the region below $\bar{\rho}=0.07$, but their actual values do not deviate considerably from the average load carrying capacity, while their exact locations appear to be very dependent on the kind of imposed waviness imperfection, suggesting that a practical exploitation of the effect of the tip radius for optimizing the beam load capacity is rather difficult.

Further insight into the conditions of buckling initiation for different notch tip radii is given in Fig. 15. The four snapshots correspond to selected points of the left hand side diagram of Fig. 14 with $\bar{\rho}=0.0254,0.0507,0.1014$ and 0.2028 , for an asymmetric waviness imperfection of maximum misalignment angle $\bar{\phi}=0.60^{\circ}$. As already observed in Fig. 14, an increased notch tip radius has only a weak effect on the critical bending load, with values of the corresponding normalized stress varying for the here considered cases from 0.0061 to 0.0067 . The weak impact of the notch tip radius is 

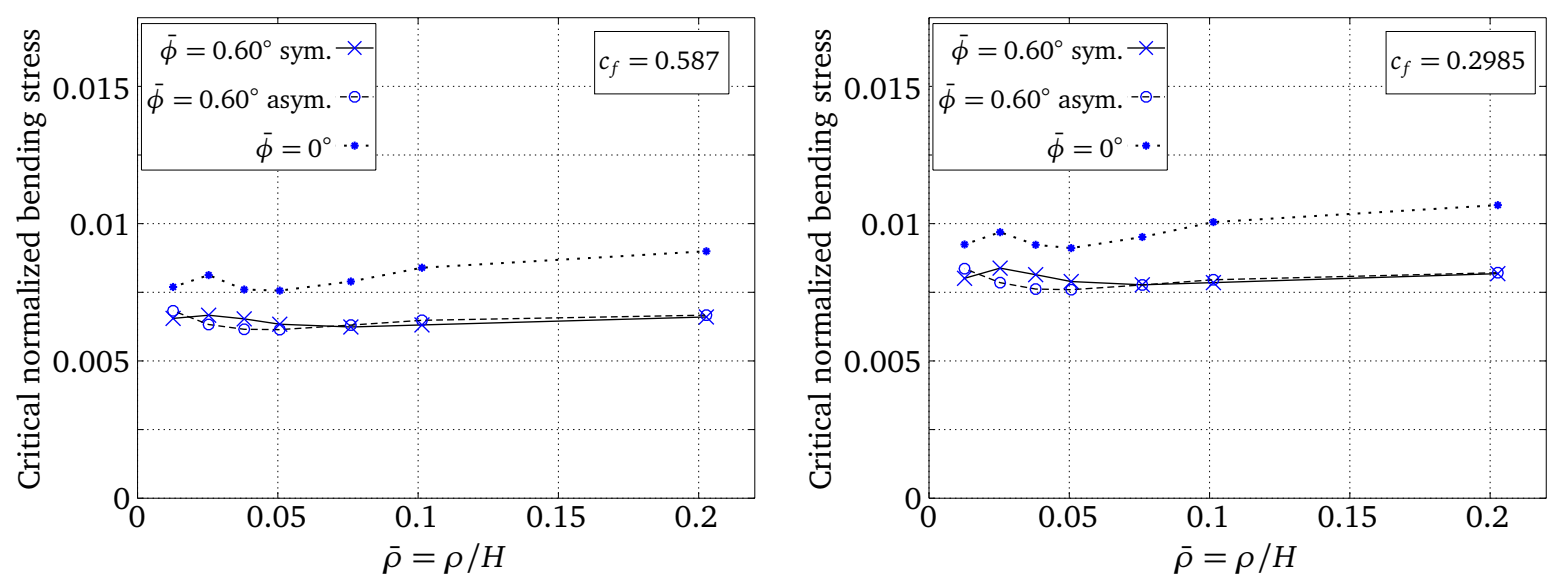

Figure 14: Critical bending load as a function of the notch tip radius $\rho\left(\right.$ for $\bar{H}_{\delta}=0.2, \bar{h}_{f}=0.0013$, $\left.\bar{p}_{w}=30\right)$.
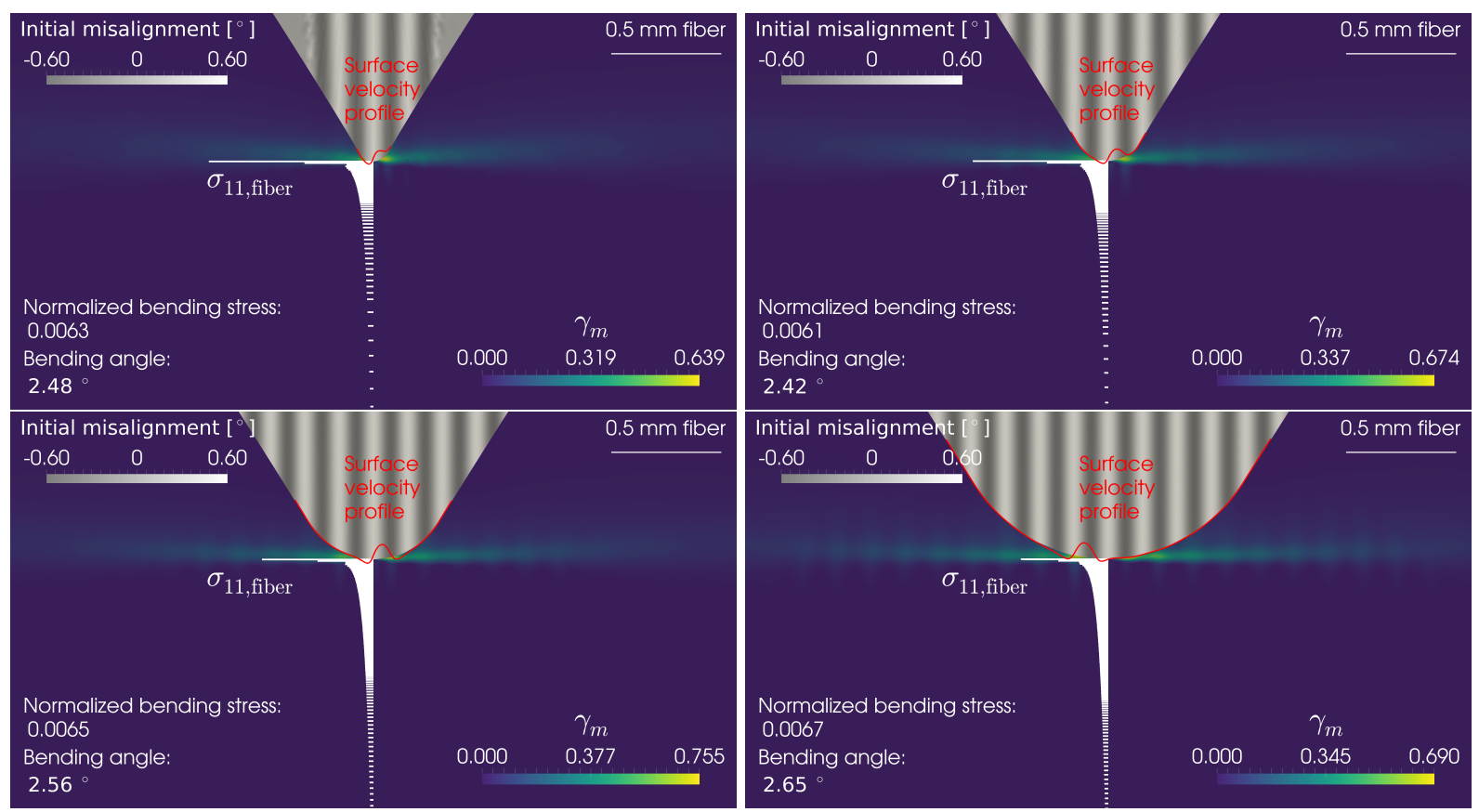

Figure 15: Micro-buckling initiation for different notch tip radii (and $\bar{H}_{\delta}=0.2, \bar{h}_{f}=0.0013, \bar{p}_{w}=30$, $\bar{\phi}=0.60^{\circ}$, asymmetric waviness imperfection).

also reflected on the maximum value of the plastic strain in the matrix material at buckling initiation, which is in the range between 0.639 and 0.755 , although the notch tip radius is varied in a range of approximately one order of magnitude.

The snapshots of Fig. 15 also include an illustration of the longitudinal stress component in the reinforcing fiber material through the cross section at the center of the notch, shown by the white horizontal lines. The illustrated stress distributions are strongly non-linear. Hence, although the critical normalized bending stresses evaluated for these examples are quite low, the actual stresses close to the top surface are very high compared to the plain beam studied in the previous section. The 
stress profiles illustrated in Fig. 15 are actually scaled with the corresponding normalized bending stresses, so that height differences among the inscribed diagrams, do not reflect differences of the absolute bending load but differences in the non-linearity of the stress distribution through the cross section. In other words, if all stress distributions were linear, the plots in the four considered cases would be identical, irrespective of the actual value of the critical bending load. This means that the higher peak values observed in the plots for lower notch tip radii correspond directly to the higher stress concentration factors expected for these cases. Nevertheless, it appears that the considerable differences in stress concentration have only a weak impact on buckling initiation. The non-local nature of buckling initiation, due to the bending stiffness of the reinforcing layers appear to make failure initiation less sensitive to highly localized stress peaks, indicating a fairly large notch support factor.

Last for this example, Fig. 16 demonstrates a very low impact of the relative notch depth $\bar{H}_{\delta}$ on the bending load capacity. Despite the different geometry, this result could possibly be inferred from Fig. 4 which corresponds to a sinus like notch imposed to the plain beam geometry of the previous section. Within the range of relatively low values of $\bar{H}_{\delta}$ covered in Fig. 4, there is already an indication that the expected critical bending load flattens out towards the upper end of the considered range. This observation is confirmed for the notched example of this section in Fig. 16, which demonstrates a constant bending load capacity above the value $\bar{H}_{\delta}=0.5$.

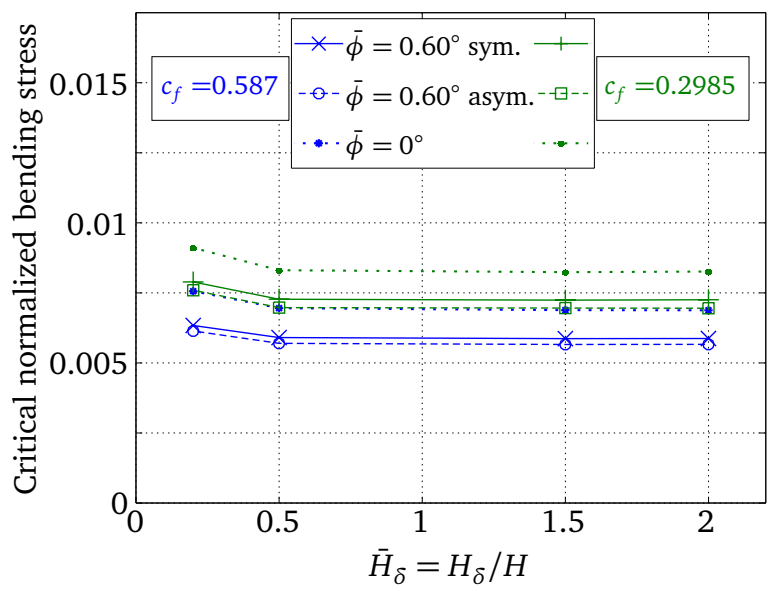

Figure 16: Critical bending load as a function of the notch depth $H_{\delta}$ (for $\bar{\rho}=0.0507, \bar{h}_{f}=0.0013$, $\left.\bar{p}_{w}=30\right)$.

It should be noted that the variants of Fig. 16 for $\bar{H}_{\delta}=2$ and $c_{f}=0.587$ correspond to the beam that was studied experimentally and numerically by Wind et al. (2015). In that work, a four point bending setup was used for introducing a bending moment and the applied vertical force at buckling initiation was measured. The average vertical force of $765 \mathrm{~N}$ reported by Wind et al. (2015), combined with a distance of $40.3 \mathrm{~mm}$ between the support points, results in a bending moment of $15415 \mathrm{~N} \mathrm{~mm}$. With a beam thickness of $T=5.9 \mathrm{~mm}$, this moment corresponds to a value of the normalized stress according to Eq. (13) equal to 0.0058. This value is lower than the critical normalized moment of 0.0069 reported in Fig. 16 for the imperfection-free case, but it is very close to the values of 0.0059 and 0.0057 respectively reported for the symmetric and asymmetric waviness imperfections of $\bar{\phi}=0.60^{\circ}$. 


\section{Compression of a sample with a circular hole}

This section is concerned with uniaxial compression along the reinforcement layering direction in the region around a circular hole. The modeled domain corresponds to the material between the considered hole circumference of a reference radius $r=1 \mathrm{~mm}$ and an external circular boundary of radius $R=20 r$. Hence, the reference radius $r$ and the ratio $R / r$ can fully define the considered geometry. Nevertheless, the latter is only of minor importance, provided that it is sufficiently large.

The layering is assumed to be nominally horizontal and two different cases are taken into account with respect to the boundary conditions. In both cases the horizontal displacements on the outer boundary of the domain are prescribed according to the far field deformation of a hole-free sample under uniaxial compression in the horizontal direction. Regarding the vertical displacement component, in the first case, zero vertical displacements are prescribed, while in the second case, a zero transverse stress condition is assumed.

The composite micro-structure is primarily characterized through the fiber volume fraction $c_{f}$ and the relative fiber height $\bar{h}_{f}=h_{f} /(2 r)$. It is additionally provided, as in the previous examples, with a waviness imperfection along the horizontal direction which is limited within a circular region of radius $1.5 r$ and characterized through the relative waviness wavelength $\bar{p}_{w}=p_{w} / h_{f}$ and the maximum misalignment angle $\bar{\phi} \approx \pi a_{w} / p_{w}$. In the numerical results to be presented in this section, a constant $c_{f}=0.6$ is considered, while $\bar{h}_{f}, \bar{p}_{w}$ and $\bar{\phi}$ are subject of parametric studies. Both symmetric and asymmetric waviness imperfections with respect to the vertical cross section passing through the hole center were tested. Due to the differences between the two being rather limited however, only results for symmetric imperfections will be presented.

In the case of symmetric imperfections, the model can be reduced to only the first quarter of the full circumference by fixing the vertical displacements of the side lying on the x-axis and the horizontal displacements of the side along the y-axis. The component of the internal kinematic variable $\boldsymbol{d}$ parallel to the layering is restricted on both aforementioned sides, so that only micro-deformation of the kind shown corresponding to Fig. 1 (c) are permitted. The same condition is applied also on the outer circumference, i.e. at radius $R$.

The material parameters for the fiber and matrix constituents correspond to a fiber-to-matrix contrast parameter $\bar{E}_{f} / \bar{E}_{m}=100$ and Poisson's ratios $v_{f}=v_{m}=0.3$, while the Ramberg-Osgood parameters for the matrix material are $\sigma_{y m} / \bar{E}_{m}=0.0315, n_{m}=4$ and $\alpha_{m}=3 / 7$.

Fig. 17 demonstrates the strong dependence between the relative fiber height $\bar{h}_{f}$ and the critical far field strain in the horizontal direction at micro-buckling initiation. For the sake of simplicity, only results for two imperfection-free cases $\left(\bar{\phi}=0^{\circ}\right)$ are included here, since corresponding results for cases with moderate waviness imperfections are very similar. The two cases included, correspond to the aforementioned two options regarding the boundary condition in the transverse direction. According to the presented curves, the investigated range of relative fiber height can be roughly split into two distinct regimes with respect to the value $\bar{h}_{f}=0.0125$. In the lower range regime, the slope of the curves is higher and there is no significant difference between the two transverse boundary conditions. The impact of the transverse boundary condition becomes considerable in the higher range regime, with the two curves remaining approximately parallel, though at a lower slope.

The occurrence of the two distinct regimes identified in Fig. 17 is explained in Fig. 18. The five included snapshots show the region of buckling initiation at the top of the hole circumference. The snapshots actually correspond to the $1^{\text {st }}, 2^{\text {nd }}, 3^{\text {rd }}, 5^{\text {th }}$ and $7^{\text {th }}$ points of Fig. 17 , with results for constrained transverse displacements shown on the left hand side and results for free transverse displacements shown on the right hand side. The red vectors illustrate the velocities at the hole surface. From top to bottom, the increased fiber bending stiffness leads to a smoothening of the velocity field corre- 


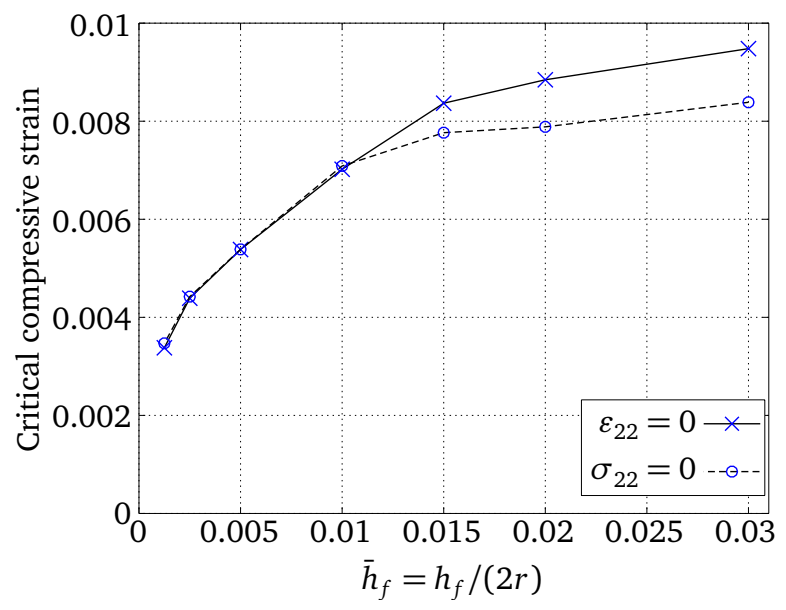

Figure 17: Critical far field compressive strain as a function of the fiber height $h_{f}\left(\right.$ for $\left.\bar{\phi}=0^{\circ}\right)$.
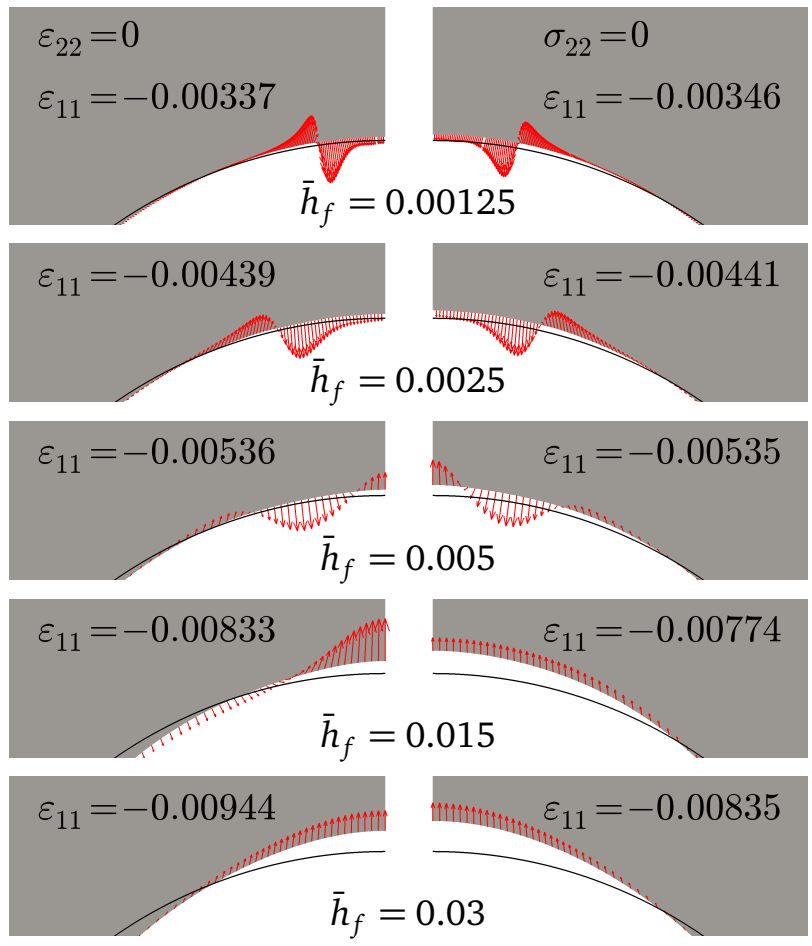

Figure 18: Micro-buckling initiation for different relative fiber heights $\bar{h}_{f}$, for constrained and free transverse displacements (left and right hand sides respectively) and for $\bar{\phi}=0^{\circ}$.

sponding to the imminent buckling mode. A common characteristic among the first three snapshots is that some portion of the hole surface moves inwards while another portion of the surface moves outwards. Moreover, the differences in the velocity profiles between the cases for a constrained or free transverse direction, are minimal.

An important transition is observed in the fourth row. While the transversely constrained case on the left hand side, still exhibits parts with inwards and outwards motion, the result for free transverse displacements only exhibits an outwards velocity. This lack of a sign change in the normal velocity 
component signalizes for the latter case a transition to a different buckling mode and at the same time the beginning of the high range regime of Fig. 17. For even larger values of the fiber height, as in the snapshots shown in the fifth row, both cases have transitioned to the buckling mode with the complete velocity profile pointing outwards. In contrast to the initial buckling mode the second one requires a net material transfer in the vertical direction. For this reason, constraining the transverse displacements on the outer boundary, provides, in this case, additional support and leads to the higher buckling limits observed in the corresponding curve of Fig. 17 .

Fig. 19 illustrates the impact of the fiber waviness wavelength $\bar{p}_{w}$ on the critical far field strain at buckling initiation. Only a weak dependence is observed with a minimum approximately at $\bar{p}_{w}=50$. For the fairly low value assumed for the fiber height, no significant differences between the cases of constrained and free transverse displacements are observed within the investigated range of fiber waviness wavelengths.

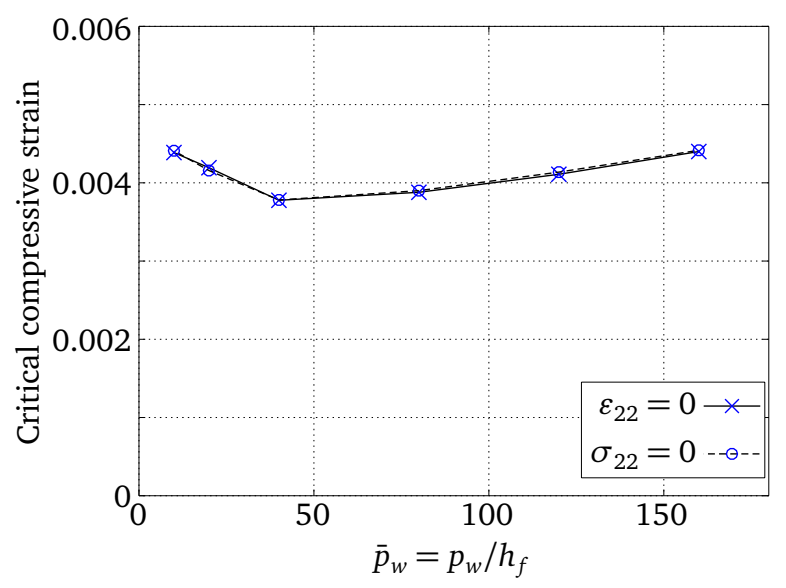

Figure 19: Critical far field compressive strain as a function of the fiber waviness wavelength $p_{w}$ (for $\bar{h}_{f}=0.0025$ and $\bar{\phi}=0.18^{\circ}$ ).

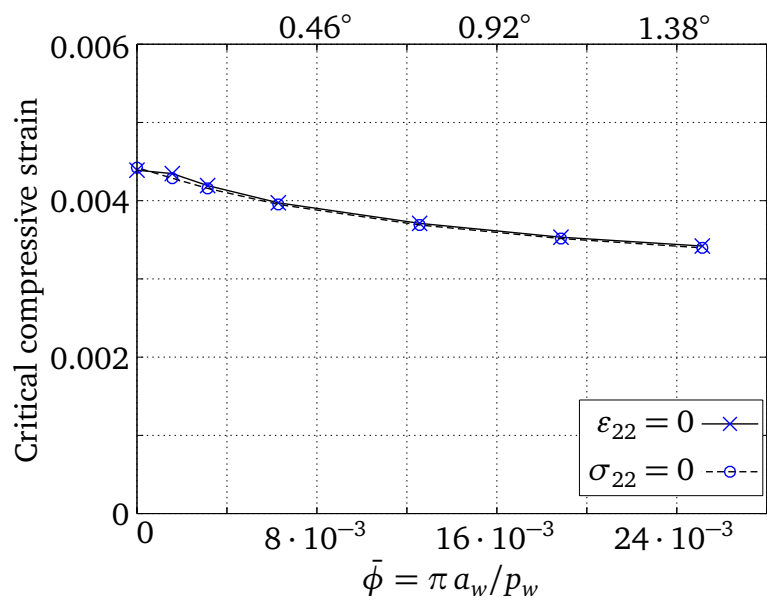

Figure 20: Critical far field compressive strain as a function of the fiber waviness amplitude $a_{w}$ (for $\bar{h}_{f}=0.0025$ and $\bar{p}_{w}=20$ ). 
The impact of the maximum fiber misalignment angle due to the imposed waviness imperfection on the critical far field strain at buckling initiation is shown in Fig. 20. As expected, the critical strain decreases with increasing fiber misalignment angle. It is however, a rather moderate dependence.

In general, this last example confirms the crucial role of the reinforcing layers bending stiffness on micro-buckling initiation. The presented numerical results also suggest a possible reinterpretation of the experimental results by Khamseh and Waas (1992), who found an increasing critical far-field strain at failure with a decreasing ratio of hole diameter to total width of the specimen. Although the diameter to width ratio captures the effect of cross sectional area reduction for larger holes precisely, for smaller holes it is the ratio of fiber diameter to hole diameter, considered here, which reflects the micro-mechanical roots of the apparent strength increase better. Moreover, the presented example demonstrates that a combination of stress concentration, size-scale effects and anisotropy results in non-trivial behaviors which are rather difficult to predict or estimate by means of analytical models, even if such models exist for each of the involved phenomena separately.

\section{Concluding remarks}

Micro-buckling in layered composite materials in the presence of stress gradients was investigated by means of a series of numerical examples. The obtained numerical results demonstrated a crucial contribution of the bending stiffness of the reinforcing layer to the compressive load capacity of all considered cases, but especially in the cases of high stress concentrations. Moreover, the negative impact of imperfections in form of a fiber misalignment was verified and quantified. However, the effect of such imperfections was comparatively less pronounced in the examples with high stress concentrations.

The plain beam bending example, presented first, included only moderate or small stress gradients. Nevertheless, it served as a means for identifying the general tendencies and for comparing with analytical solutions for uniform loading. It also demonstrated a conical double kink-band formation in the layered micro-structure as the main post-buckling deformation pattern.

In the second example corresponding to a V-notched beam, the effect of the fiber height on the bending load capacity was more pronounced than the effect of the fiber misalignment angle. A parametric study with respect to the notch tip radius showed a weak impact on the bending load capacity, despite the fact that a significant variation of the stress concentration factor was found in the numerical results. This is an indication of a large notch support factor, attributed to the bending stiffness of the reinforcing layers and the consequent non-local material behavior.

The last example investigated the case of uniaxial compression parallel to the reinforcement, applied to the region around a circular hole. It showed, in general, the same tendencies as the results for the V-notched beam. Apart from demonstrating the impact of the fiber height on the critical load, the buckling forms for different fiber heights were presented and two distinct regimes were identified, with only the second one being affected by the kind of boundary condition transverse to the loading direction.

The complexity of some of the demonstrated phenomena, arising as a combination of several competitive mechanisms, underlines the importance of numerical tools for reaching concrete quantitative conclusions. In that sense, the here utilized homogenized finite-element model seems to be a good compromise in terms of capturing the micro-mechanical mechanisms and avoiding prohibitively large computational models. Future extensions of the underlying homogenization method to include effects such as matrix cracking, fiber-matrix splitting and fiber breakage would allow to cover a much wider range of effects with relevance for practical applications. 


\section{Acknowledgements}

This work was supported by the Danish Council for Independent Research, through the Sapere Aude project "Higher Order Theories in Solid Mechanics".

\section{References}

Aboudi, J. and Gilat, R. (2006). Buckling analysis of fibers in composite materials by wave propagation analogy. International Journal of Solids and Structures, 43(17):5168 - 5181.

Ahn, J. and Waas, A. M. (2002). Prediction of compressive failure in laminated composites at room and elevated temperature. AIAA Journal, 40(2):346-358.

Bažant, Z. P., Kim, J.-J. H., Daniel, I. M., Becq-Giraudon, E., and Zi, G. (1999). Size effect on compression strength of fiber composites failing by kink band propagation. International Journal of Fracture, 95(1-4):103-141.

Budiansky, B. (1983). Micromechanics. Computers \& Structures, 16(1-4):3 - 12.

Budiansky, B. and Fleck, N. A. (1993). Compressive failure of fiber composites. Journal of the Mechanics and Physics of Solids, 41(1):183-211.

Davidson, P., Pineda, E. J., Heinrich, C., and Waas, A. M. (2013). A unified model for predicting the open hole tensile and compressive strengths of composite laminates for aerospace applications. 54th AIAA Structures, Structural Dynamics, and Materials Conference.

Fleck, N. A. (1997). Compressive failure of fiber composites. Advances in Applied Mechanics, 33:43117.

Fleck, N. A. and Shu, J. Y. (1995). Microbuckle initiation in fibre composites: a finite element study. Journal of the Mechanics and Physics of Solids, 43(12):1887-1918.

Jensen, H. M. and Christoffersen, J. (1997). Kink band formation in fiber reinforced materials. Journal of the Mechanics and Physics of Solids, 45(7):1121-1136.

Khamseh, A. R. and Waas, A. M. (1992). Failure mechanisms of uni-ply composite plates with a circular hole under static compressive loading. Journal of Engineering Materials and Technology, 114(3):304.

Kyriakides, S., Perry, E. J., and Liechti, K. M. (1994). Instability and failure of fiber composites in compression. Applied Mechanics Reviews, 47(6):S262-S268.

Lee, S. H. and Waas, A. M. (1999). Compressive response and failure of fiber reinforced unidirectional composites. International Journal of Fracture, 100(3):275-306.

Ligursky, T. and Renard, Y. (2014). A continuation problem for computing solutions of discretised evolution problems with application to plane quasi-static contact problems with friction. Computer Methods in Applied Mechanics and Engineering, 280:222-262.

Liu, G., Thouless, M. D., Deshpande, V. S., and Fleck, N. A. (2014). Collapse of a composite beam made from ultra high molecular-weight polyethylene fibres. Journal of the Mechanics and Physics of Solids, 63:320 - 335. 
Parnes, R. and Chiskis, A. (2002). Buckling of nano-fibre reinforced composites: a re-examination of elastic buckling. Journal of the Mechanics and Physics of Solids, 50(4):855 - 879.

Pinho, S. T., Gutkin, R., Pimenta, S., De Carvalho, N. V., and Robinson, P. (2012). On longitudinal compressive failure of carbon-fibre-reinforced polymer: from unidirectional to woven, and from virgin to recycled. Philosophical Transactions of the Royal Society A, 370(1965):1871-1895.

Poulios, K. and Niordson, C. F. (2016). Homogenization of long fiber reinforced composites including fiber bending effects. Journal of the Mechanics and Physics of Solids, 94:433-452.

Prabhakar, P. and Waas, A. M. (2013). Interaction between kinking and splitting in the compressive failure of unidirectional fiber reinforced laminated composites. Composite Structures, 98:85.

Rosen, B. W. (1965). Mechanics of composite strengthening. In Fiber Composite Materials. American Society for Metals, Metals Park, Ohio.

Soutis, C. and Fleck, N. A. (1990). Static compression failure of carbon fibre T800/924C composite plate with a single hole. Journal of Composite Materials, 24(5):536-558.

Soutis, C., Fleck, N. A., and Smith, P. A. (1991). Failure prediction technique for compression loaded carbon fiber-epoxy laminate with open holes. Journal of Composite Materials, 25(11):1476-1498.

Waas, A. M., Ahn, J., and Khamseh, A. R. (1998). Compressive failure of notched uniply composite laminates. Composites Part B, 29(1):75-80.

Waas, A. M., Babcock, C. D., and Knauss, W. G. (1990). A mechanical model for elastic fiber microbuckling. Transactions of the ASME. Journal of Applied Mechanics, 57(1):138-149.

Wind, J. L., Waas, A. M., and Jensen, H. M. (2015). Initiation of failure at notches in unidirectional fiber composites. Composite Structures, 122:51 - 56. 